\title{
Heart
}

\section{Updated guidance from NICE on implantable defibrillators: does it work in real life?}

\begin{tabular}{|r|l|}
\hline Journal: & Heart \\
\hline Manuscript ID & Draft \\
\hline Article Type: & Editorial \\
\hline Date Submitted by the Author: & $\mathrm{n} / \mathrm{a}$ \\
\hline Complete List of Authors: & Cowie, Martin; Imperial College London, National Heart \& Lung Institute \\
\hline & $\begin{array}{l}\text { Implanted cardiac defibrillators < Electrophysiology < CARDIAC } \\
\text { PROCEDURES AND THERAPY, Heart failure }<\text { DISEASES, Ventricular } \\
\text { fibrillation < Cardiac arrhythmias and resuscitation science }<\text { DISEASES, } \\
\text { Ventricular tachycardia < Cardiac arrhythmias and resuscitation science }< \\
\text { DISEASES }\end{array}$ \\
\hline & \multicolumn{2}{|l}{} \\
\hline
\end{tabular}

SCHOLARONE ${ }^{m}$

Manuscripts 


\section{Updated guidance from NICE on implantable defibrillators: does it work in real life?}

The National Institute for Health and Clinical Excellence (NICE) was set up (under a slightly different name) in 1999 as a public body independent of the UK Government, with the remit to reduce the variation in the availability and quality of NHS treatments and care in England. It has published numerous clinical guidelines and technology appraisals, and is well respected around the world for its robust and transparent assessment of the evidence, and the opportunity it gives relevant stakeholders to input to the assessment process. In June 2014 it published its $314^{\text {th }}$ technology appraisal (TA314), ${ }^{1}$ updating its guidance on the use of implantable defibrillator (ICD) technology, and merging this with an update on its guidance on the use of cardiac resynchronisation technology (CRT).

In the absence of new trial evidence, TA314 made the same recommendations for the secondary prevention of sudden cardiac death as in its previous guidance (TA95, January 2006): ICDs continued to be recommended for people who had survived a cardiac arrest caused by either ventricular tachycardia (VT) or ventricular fibrillation, or had spontaneous sustained VT causing syncope or significant haemodynamic compromise, or had sustained VT without syncope or cardiac arrest and a left ventricular ejection fraction (LVEF) $\leq 35 \%$ and symptoms no worse than NYHA Class III. Those with a familial cardiac condition with a high risk of sudden death (such as long QT Syndrome, Brugada Syndrome, hypertrophic cardiomyopathy, or arrhythmogenic right ventricular dysplasia) or surgically repaired congenital heart disease also continued to be included in the recommendations as candidates for an ICD.

In contrast, there were major changes in the guidance related to the primary prevention of sudden death. The guidance was no longer limited to ischaemic cardiomyopathy, and the use of Holter monitoring and electrophysiological studies to test the inducibility of VT disappeared. Emphasis was now focused on all patients with an EF $\leq 35 \%$, and eligibility for an ICD or CRT-D/CRT-P implantation was largely determined by QRS duration and morphology, and by NYHA class. A simple Table (Table 1) was provided to illustrate which technologies were optimal for which patients based on these characteristics.

This approach was based on individual patient data (IPD) synthesised by network meta-analysis from 13 randomised trials (12 638 patients) provided by medical technology companies - representing $95 \%$ of patients enrolled in randomised controlled trials of such implantable devices. ${ }^{2}$ These data provided estimates for expected relative benefit conditional upon multiple patient characteristics, and likely cost-effectiveness based on the absolute levels of mortality, hospitalisation and quality of life reported in the trials. NICE decided to accept the importance of QRS duration and left bundle branch block, but dropped the evidence that gender and age affected the relative benefit.

Cubbon et al, in this edition of Heart, ${ }^{3}$ set out to determine how well the new NICE guidance works in real life - does it identify the individuals with increased risk of sudden cardiac death? They constructed a historical cohort of 1091 patients with heart failure with reduced ejection fraction (HFREF) that had been prospectively identified in several cardiology outpatient departments in the UK between June 2006 and December 2011, and followed up for a mean of 3.7 years. The mean age of these patients was 68 and $74 \%$ were male, $63 \%$ had ischaemic heart disease and $26 \%$ were diabetic. $47 \%$ had a QRS duration of at least 120 ms (of these: $29 \%$ had LBBB, $6 \%$ RBBB and $12 \%$ a non-specific morphology). The use of heart failure drug therapy was high - $89 \%$ were on an ACE inhibitor or angiotensin receptor blocker, $81 \%$ were on a beta-adrenoreceptor blocker, and $41 \%$ on a 
mineralocorticoid receptor antagonist - and 31\% had an implantable device (3\% ICD alone, 18\% CRT$\mathrm{P}$, and $9 \%$ CRT-D). 344 patient died or had an appropriate ICD shock during the follow-up period (9 events per 100 person years), and 78 of these events ( 2 events per 100 person years) were classified as sudden cardiac deaths (SCD) including 50 deaths and 28 appropriate ICD shocks ( 8 for VF, 20 for VT with a median programmed threshold for ICD shock of 188bpm).

Within this hospital-based cohort, 31\% fulfilled the specific NICE criteria for an ICD given in Table 1 (i.e. QRS duration $\geq 120 \mathrm{~ms}$, NYHA Class $1-3$, and LVEF $\leq 35 \%$ ). Such patients had a substantially increase risk of SCD (Hazard ratio [HR] 2.5 (95\% confidence interval 1.6-3.9) $p<0.001$ ) and a somewhat increased risk also of progressive pump failure death (HR 1.6 (1.1-2.3) $p=0.02$ ), and noncardiovascular death (HR $1.5(1.1-2.2) p=0.027)$. This is perhaps not surprising - such patients were older and had more impaired LVEF than those who did not fulfil the NICE criteria. However, Cubbon et al also clearly show that within their study population, the absolute risk of SCD was heavily influenced by the presence of diabetes mellitus and ischaemic heart disease - so the event rates in patients with diabetes (or ischaemic heart disease) not meeting the specific TA314 criteria (due to a narrow QRS complex) were similar to patients without diabetes (or ischaemic heart disease) who did. This highlights a flaw in the NICE guidance - although the recommended approach works well to identify patients at high relative risk of SCD, it does not provide a precise estimate of an individual's absolute risk, and presumably, therefore, the likely absolute benefit. If the absolute benefit is not considered then not only may the physician and patient have an unrealistic estimate of the potential benefit of ICD technology, but NHS resources may be used inappropriately.

The 'blind' application of Table 1 from the guideline could lead to an oversimplified approach with the risk of over-treatment of some patients, and under-treatment of others, and marked variation from one centre to another. TA314 recognises this, with the Appraisal Committee stating that "careful, explicit and shared decision-making about appropriate use of these technologies...is important" although "preventing sudden cardiac death.... is challenging....and there is currently no optimal strategy for risk stratification". ${ }^{1}$ Marrying detailed IPD meta-analysis from the clinical trials, with real world data relating to the background level of risk, is not easy nor is it easy to communicate the risks and benefits in an appropriate way to patients who may not know what questions to ask. Cubbon's work suggests that patients with HFREF seen in a NHS cardiology clinic have an annual risk of sudden cardiac death, despite optimal medical therapy, that is substantial around $2 \%$ - so ensuring risk stratification takes place is vital.

It is now widely recognised that patients should be active partners in decisions about their healthcare. Despite considerable support from NICE and other professional bodies for such shared decision making, ${ }^{4} \mathrm{NHS}$ surveys consistently report that at least $40 \%$ of patients want to be more involved in decisions about their care, and $20 \%$ report that they were given insufficient information about their treatment. ${ }^{5}$ Key questions include: What are my options? What are the benefits and possible harms? How likely are these? The new NICE guidance on ICDs goes some way in helping clinicians to use the evidence-base to determine (and discuss) the best option for the individual patient, but much further work is needed before we are in the situation where advice can be accurately tailored to the individual, and where shared decisions can be meaningfully made.

What is clear is that the implementation of TA314 should lead to an increase in the implant rate for 'high energy' devices. Of the 1000 or so patients with HF seen in the outpatient clinics by Cubbon et al, at least 30\% (and perhaps as high as 60\% depending on how 'high risk' is defined in those with a QRS duration < $120 \mathrm{~ms}$ ) of patients are potentially eligible for an ICD. Similar reports have appeared from other centres. ${ }^{6}$ Currently, English implant rates are well below the Western European average, but the rates appear to be steadily increasing despite major financial strains on the NHS. ${ }^{7}$ The 
official NICE assessment of the cost implications of implementing TA314 in England was $119 \mathrm{~m}$ in the first year, and $£ 33 \mathrm{~m}$ in the second and subsequent years, although it accepted that implant rates varied 'considerably' across the country. ${ }^{8}$ Such variation is something that NICE is charged with reducing.

Ensuring shared decision making based on best available trial data combined with real-life evidence and clinical expertise is a tall order, but something that all stakeholders should pursue without delay. Only then will there be more equitable implementation of the guidance and a reduction in the huge variation in practice seen across the UK in the identification of patients with HFREF for whom an ICD may be appropriate as part of a strategy to reduce the risk of sudden cardiac death.

\section{References}

1. NICE Technology appraisal guidance 314. Implantable cardioverter defibrillators and cardiac resynchronisation therapy for arrhythmias and heart failure (review of TA95 and TA120). National Institute for Health and Care Excellence, June 2014. Available at https://www.nice.org.uk/guidance/ta314/resources/implantable-cardioverter-defibrillatorsand-cardiac-resynchronisation-therapy-for-arrhythmias-and-heart-failure-review-of-ta95and-ta120-82602426443461 (Last accessed 6 January 2016).

2. Woods B, Hawkins N, Mealing S et al. Individual patient data network meta-analysis of mortality effects of implantable cardiac devices. Heart 2015; 101: 1800-6.

3. Cubbon RM, Witte K, Kearney LC et al. Performance of 2014 NICE defibrillator implantation guidelines in heart failure risk stratification. Heart 2016 (in press)

4. Shared Decision Making Programme. Measuring shared decision making: a review of research evidence. December 2012. Available at http://www.rightcare.nhs.uk/wpcontent/uploads/2012/12/Measuring Shared Decision Making_Dec12.pdf (last accessed 6 January 2016).

5. National results from the 2014 inpatient survey. National NHS patient survey programme, May 2015. Available at http://www.cqc.org.uk/sites/default/files/201500519\%20NHS\%20Inpatient\%20Survey\%202 014\%20National\%20summary\%20and\%20results\%20tables\%20FINAL.pdf (Last accessed 6 January 2016).

6. Mahendiran T, Gosling OE, Newton J et al. Latest NICE guidelines on CRT and ICD devices in heart failure may significantly increase implant rates. Br J Cardiol 2015; 22: 155.

7. National Audit of Cardiac Rhythm Management Devices 2014-15, available at www.ucl.ac.uk/nicor/audits/cardiacrhythmmanagement/publicreports (last accessed 6 January 2016).

8. NICE, June 2014. Costing statement: implementing the NICE guidance on implantable cardioverter defibrillators and cardiac resynchronisation therapy for arrhythmias and heart failure (TA314). Available at: https://www.nice.org.uk/guidance/ta314/resources/costingstatement-426950317 (last accessed 6 January 2016) 
Table 1 Treatment options with ICD or CRT for people with heart failure who have left ventricular dysfunction with an LVEF of $35 \%$ or less (according to NYHA class, QRS duration and presence of LBBB)

| From TA314 (reference 1).

\begin{tabular}{|c|c|c|c|c|}
\hline \multirow[b]{2}{*}{ QRS interval } & \multicolumn{4}{|c|}{ NYHA class } \\
\hline & I & II & III & IV \\
\hline$<120$ milliseconds & \multicolumn{3}{|c|}{$\begin{array}{l}\text { ICD if there is a high risk of } \\
\text { sudden cardiac death }\end{array}$} & $\begin{array}{l}\text { ICD and CRT not } \\
\text { clinically indicated }\end{array}$ \\
\hline $\begin{array}{l}\text { 120-149 milliseconds } \\
\text { without LBBB }\end{array}$ & $I C D$ & ICD & ICD & CRT-P \\
\hline $\begin{array}{l}\text { 120-149 milliseconds with } \\
\text { LBBB }\end{array}$ & ICD & CRT-D & CRT-P or CRT-D & CRT-P \\
\hline $\begin{array}{l}\geq 150 \text { milliseconds with or } \\
\text { without LBBB }\end{array}$ & CRT-D & CRT-D & CRT-P or CRT-D & CRT-P \\
\hline
\end{tabular}

https://mc.manuscriptcentral.com/heart 